\title{
LA EXPLICACIÓN TEÓRICA \\ DE LA POTENCIALIDAD TURÍSTICA DEL MEDIO NATURAL. VERIFICACIÓN EN ARAGÓN DEL MODELO DE WARSZYNSKA A PARTIR DEL ANÁLISIS FACTORIAL
}

\author{
Javier CALLIZO SONEIRO y Antonio J. LACOSTA ARAGÜÉS \\ Departamento de Geografía y Ordenación del Territorio \\ Universidad de Zaragoza
}

\begin{abstract}
Resumen: Tomando el caso concreto del espacio turístico de Aragón, los autores han querido verificar la bondad del modelo teórico de Warszynska mediante la utilización de la técnica factorial. La cuestión central es la siguiente: ¿qué parte de la oferta turística es explicable a partir de las variables independientes - agua, altitud, cubierta forestal - seleccionadas por la geógrafa polaca? El análisis factorial permite descubrir la existencia de lógicas espaciales diferentes y aun contrastadas entre el turismo convencional y las formulas de tipo sostenible.
\end{abstract}

Palabras claves: Aragón, turismo sostenible, potencialidad, medio ambiente, oferta turística, Warszynska.

\begin{abstract}
Taking the concrete case of the tourist space of Aragon, in this paper the authors have wanted to verify the excellence of the theoretical model of Warszynska through the utilization of the factorial technique. The central issue is as follows: what part of the tourist offer is explainable in terms of the independent variables (water, altitude, forest cover) selected by the Polish geographer? The factor analysis allows us to discover the existence of different and even contrasted kinds of spatial logic among conventional and sustainable tourism formulations.
\end{abstract}

Key Words: Aragon, sustainable tourism, potential pattern, natural environnment, tourist offer, Warszynska.

De los múltiples intentos de explicación científica del potencial turístico, 1 a mayoría se refiere sobre todo al clima, elemento, éste, muy vinculado al viaje turístico desde los primeros momentos. En los trabajos de CLAUSSE y GUÉROUT (1955), POULTER (1962), HUGHES (1967), DAVIS (1968), FLOCAS (1975), DAUPHINÉ y GHILARDI (1978), SARRAMÉA (1980), MIECZKOWSKI (1985) y MARCHAND (1986), la potencialidad climática de un determinado lugar es explicada mediante una regresión múltiple a partir de la temperatura ( $\left.x_{1}\right)$, la insolación ( $\left.x_{2}\right)$ y las 
precipitaciones ( $x_{3}$ ), del tipo: $y=a+b x_{1}+c x_{2}-d x_{3}$; en el caso de Mieczkowski además los coeficientes han sido ponderados en función del porcentaje explicado por cada una de las variables independientes (el valor estimado de y oscilará entonces entre 0 y 100).

Entre los primeros intentos de explicación global de la atracción turística del cuadro natural brilla con luz propia la geógrafa polaca Warszynska (1974).

El índice de atracción se formula así:

$$
I P M N_{i j}=\sum_{i=1}^{3}\left(\frac{S_{i j}}{S t_{i j}}\right)^{\left[\left(\frac{1}{l_{j}}\right) \cdot\left(\frac{\bar{S}_{j}}{S_{i j}}\right)\right]}
$$

Siendo:

$I P M N_{i j}$ : Indice de potencial turístico del medio natural, obtenido de la suma de los coeficientes de atracción calculados para las tres variables j consideradas en el municipio i (cubierta forestal, superficie de la lámina de agua, altitud).

$S_{i j}$ : Superficie de la variable j en ei municipio i.

$S t_{i j}$ : Superficie total del municipio i.

$\bar{S}_{j}$ : Superficie media por municipio de la variable $\mathrm{j}_{r}$ para el conjunto de 1 a región.

$l_{j}$ : Grado de atracción de cada variable j.

En definitiva, el modelo se sustenta en dos pilares: a) una ponderación de las diferentes variables, obtenida empíricamente de acuerdo con las preferencias de la clientela; y b) la superficie ocupada por cada una de las variables, medida en términos relativos según los valores regionales.

La ecuación de Warszynska se hace eco así del fuerte tropismo de las sociedades europeas hacia la altitud - al menos hasta los años ochenta-, al tiempo que de la 
atracción selectiva de las masas forestales, según su calidad y estado de conservación, y de los recursos hídricos como remedo interior del tropismo litoral.

La aplicación concreta al caso de Aragón (mapa 1) ha sido hecha a partir de las variables altitud, masa forestal y superficie de la lámina de agua disponible para usos potencialmente recreativos; los coeficientes $l_{j}$, de acuerdo con las recomendaciones de Warszynska, ponderaron las variables del siguiente modo:

a) altitud: 0,5 hasta $300 \mathrm{~m} ; 1$ de 300 a $600 \mathrm{~m} ; 2$ de 600 a $900 \mathrm{~m} ; 3$ de 900 a $1.200 \mathrm{~m}$; 4,5 por encima de $1.200 \mathrm{~m}$,

b) masa forestal: 4,5 para el bosque maderable; 3 para el bosque aclarado; 1 para el monte leñoso.

c) lámina de agua: 5 .

Pues bien, los cinco municipios aragoneses que se sitúan a la cabeza de la atracción estimada albergan algunos de los enclaves de mayor atracción turística observada: Benasque, La Fueva, Montanuy, Biescas y Valle de Hecho. En los primeros puestos aparece también Zaragoza (2'543) gracias, ya que no a la altitud, al enorme potencial de su lámina de agua (el Ebro, el Canal Imperial y los galachos de Juslibol y La Alfranca) así como a la cubierta vegetal a aquélla asociada; y unos pocos puestos después lo hace Caspe, que, con 2'095, debe al embalse de Mequinenza su elevada atracción potencial.

Las serranías pirenaicas e ibéricas acaparan finalmente la mayor parte de la potencialidad recreativa estimada. La mayor parte, pero no toda: las láminas de agua - de cursos fluviales y en especial de lagos y embalses- añaden un plus de atracción a los espacios montanos pero consiguen también ganar para el turismo enclaves de la tierra llana vedados a la actividad turística por lo tórrido de su canícula.

La ponderación de Warszynska parece reflejar muy bien, al menos en apariencia, lo que el sentido común entiende por espacio potencialmente recreativo. Ahora bien, ¿cuál es exactamente el lazo causal entre la localización de la oferta turística y el peso de las variables ambientales señaladas por Warszynska? ¿Lo hay acaso? 


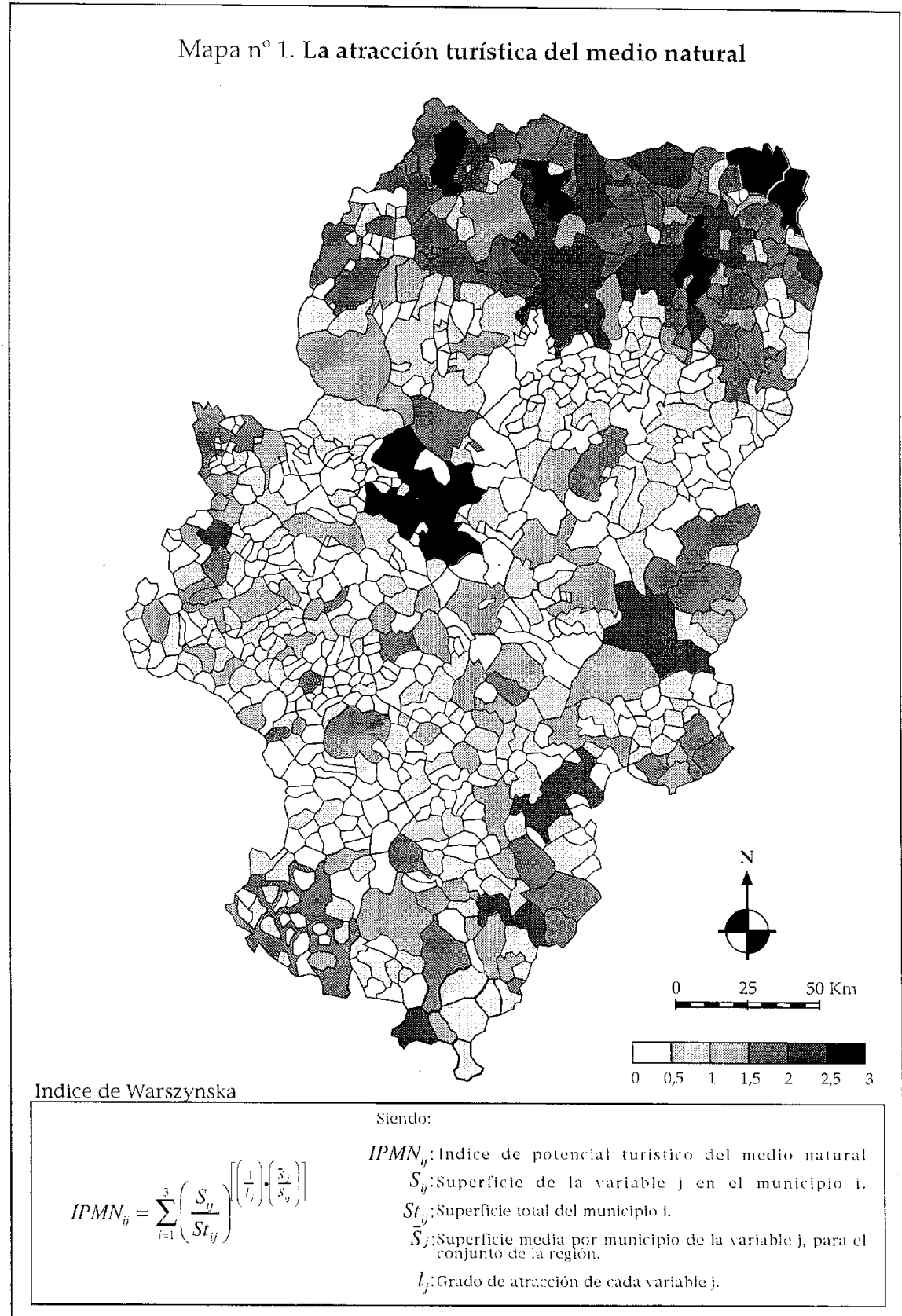

FUENTE: «Distribución general de tierras por municipios, ano 1991». Bolctin de hiticadores Estadisticos de Aractiol. N"15, Ill trimestre, 1992. ELABORACION: Callizo Soneiro, J. y Lacosta Aragües, A. 
Las ecuaciones de regresión múltiple nos proporciona la medida de esa relación: como mucho, las variables causales o independientes consideradas no explican más allá del 25 por 100 de la variancia total de la variable dependiente, en este caso la tasa de función turística como expresión del peso relativo de la oferta; del resto, otro tanto al menos no es explicable sino mediante el protagonismo de los agentes económicos y sociales (endógenos y exógenos, por supuesto), y queda otra parte de la oferta -no inferior al 30 por 100, estimamos- en cuya explicación las variables relacionadas con el patrimonio cultural tienen mucho que decir. Dicho de otra manera: por muy alta que sea la cifra estimada del potencial recreativo de un determinado lugar, la conversión de éste en espacio turístico dependerá decisivamente de la voluntad de los agentes económico-sociales.

El intento de descubrir la lógica espacial que subyace en el mapa de la localización de la oferta nos ha llevado hasta la técnica factorial. En este caso, se ha aplicado a 726 municipios aragoneses (todos excepto tres, de los que no había datos), considerando diez variables cuya matriz de correlaciones es la siguiente:

Tabla 1. Matriz de correlaciones

\begin{tabular}{|c|c|c|c|c|c|c|c|c|c|c|}
\hline Variable & $\begin{array}{c}\text { Altitud } \\
\text { m }\end{array}$ & $\begin{array}{l}\text { Residencia } \\
\text { Secundaria }\end{array}$ & $\begin{array}{l}\text { Hoteles y } \\
\text { hostales }\end{array}$ & Pensiones & Camping & $\begin{array}{c}\text { Vivienda } \\
\text { de turismo } \\
\text { rural } \\
\text { VTR } \\
\end{array}$ & $\begin{array}{c}\text { Monte } \\
\text { maderable }\end{array}$ & $\begin{array}{l}\text { Monte } \\
\text { abierto }\end{array}$ & $\begin{array}{l}\text { Monte } \\
\text { leñoso }\end{array}$ & $\begin{array}{l}\text { Ríos y } \\
\text { lagos }\end{array}$ \\
\hline Altitud m & 1 & 0,002 & 0,033 & $-0,003$ & 0,165 & 0,2 & 0,263 & 0,138 & 0,101 & $-0,078$ \\
\hline $\begin{array}{l}\text { Residencia } \\
\text { Secundaria }\end{array}$ & 0,002 & 1 & 0,93 & 0,88 & 0,251 & 0,05 & 0,2 & 0,096 & 0,15 & 0,278 \\
\hline $\begin{array}{l}\text { Hoteles y } \\
\text { hostales }\end{array}$ & 0,033 & 0,93 & 1 & 0,92 & 0,31 & 0,081 & 0,17 & 0,098 & 0,109 & 0,32 \\
\hline Pensiones & $-0,003$ & 0,88 & 0,92 & 1. & 0,229 & 0,074 & 0,162 & 0,063 & 0,118 & 0,31 \\
\hline Camping & 0,165 & 0,251 & 0,31 & 0,229 & 1 & 0,399 & 0,461 & 0,223 & 0,295 & 0,25 \\
\hline $\begin{array}{l}\text { Vivda. Tur. } \\
\text { rural VTR }\end{array}$ & 0,3 & 0,05 & 0,081 & 0,074 & 0,399 & 1 & 0,459 & 0,158 & 0,306 & 0,214 \\
\hline $\begin{array}{l}\text { Monte } \\
\text { maderable }\end{array}$ & 0.263 & 0,2 & 0,17 & 0,162 & 0,461 & 0,459 & 1. & 0,414 & 0,565 & 0,316 \\
\hline $\begin{array}{l}\text { Monte } \\
\text { abierto }\end{array}$ & 0,138 & 0,096 & 0,098 & 0,063 & 0,223 & 0,158 & 0,414 & 1 & 0,331 & 0,184 \\
\hline $\begin{array}{l}\text { Monte } \\
\text { leñoso }\end{array}$ & 0,101 & 0,15 & 0,109 & 0,118 & 0,295 & 0,306 & 0,565 & 0,331 & 1 & 0,42 \\
\hline Ríos y lagos & $-0,078$ & 0,278 & 0,32 & 0,31 & 0,25 & 0,214 & 0,316 & 0,184 & 0,42 & 1 \\
\hline
\end{tabular}

Tras la extracción factorial mediante el método de componentes principales, se procedió a su rotación con Varinax, obteniéndose la factorización que recoge la tabla 2. 
Tabla 2. Factorización de las variables*

\begin{tabular}{|c|c|c|c|c|c|c|c|c|c|c|}
\hline Variable & $\underset{\mathrm{m}}{\text { Nititud }}$ & $\begin{array}{l}\text { Rescia. } \\
\text { Secundaria }\end{array}$ & $\begin{array}{l}\text { Hoteles y } \\
\text { hostales }\end{array}$ & Pensiones & Camping & $\begin{array}{l}\text { Viv. Tur. } \\
\text { rural } \\
\text { VTR }\end{array}$ & $\begin{array}{c}\text { Monte } \\
\text { maderable }\end{array}$ & $\begin{array}{l}\text { Monte } \\
\text { abierto }\end{array}$ & $\begin{array}{l}\text { Monte } \\
\text { leñoso }\end{array}$ & $\begin{array}{l}\text { Ríos y } \\
\text { lagos }\end{array}$ \\
\hline Factor I & 0,029 & 0,959 & 0,979 & 0,958 & 0,175 & $-0,085$ & $-0,001$ & $-0,007$ & $-0,091$ & 0,158 \\
\hline Factor II & 0,486 & $-0,002$ & 0,044 & 0,015 & 0,682 & 0,893 & 0,588 & $-0,077$ & 0,389 & 0,278 \\
\hline
\end{tabular}

*Extracción: Método de Componentes Principales; Rotación: Varimax

Los dos primeros factores, los únicos con capacidad de explicación (juntos dan cuenta del 57 por 100 de la variancia), son el producto de las siguientes correlaciones:

-El Factor I (mapa 2) debe ser interpretado como una medida del alojamiento convencional. Tal es el significado de la altísima correlación existente entre hostelería, residencia secundaria y pensiones; las tres muestran una muy escasa relación con la variable camping, siendo negativa además con las viviendas de turismo rural (V.T.R.) así como con la mayoría de las variables referidas a la calidad de la cubierta forestal; ambas tres son finalmente indiferentes al peso de la variable altitud (perturbada por la magnitud del parque turístico de la ciudad de Zaragoza) y guardan una vaga relación con la existencia de láminas de agua.

Es decir, la oferta convencional descansa fundamentalmente sobre la residencia secundaria -una demanda urbana de proximidad indiferente a la altitud-, pero también sobre una suma de factores entre los que es preciso subrayar los deportes de invierno y la demanda de hostelería vinculada con los ejes de carreteras; una oferta que es el resultado de una estratificación histórica de alcance y sentido diferente y hasta contrapuesto en algunas de sus etapas, de modo que la tendencia claramente hipsotrópica de los primeros momentos se ve desdibujada por la localización, en ámbitos altitudinales medios, de las nuevas actividades recreativas.

-El Factor II (mapa 3) no puede ser interpretado sino como el reflejo de un turismo verde, rural, sostenible en suma. Aparece ahora nítida una estrecha correlación entre la vivienda de turismo rural, el camping, la altitud, la calidad y la cantidad de la masa forestal, y es mayor que en el caso anterior la atracción ejercida por las láminas de agua; una lógica espacial que se revela indiferente a la localización de la hostelería convencional. Estamos, en puridad, no ante un destino turístico alternativo la relación es muy poco significativa pero no inversa- sino ante un "producto" turístico alternativo que en muchos casos puede compartir -y de hecho comparte- escenografía con los productos convencionales. Tipología del alojamiento y factores medioambientales están ahora estrechamente interrelacionados. 


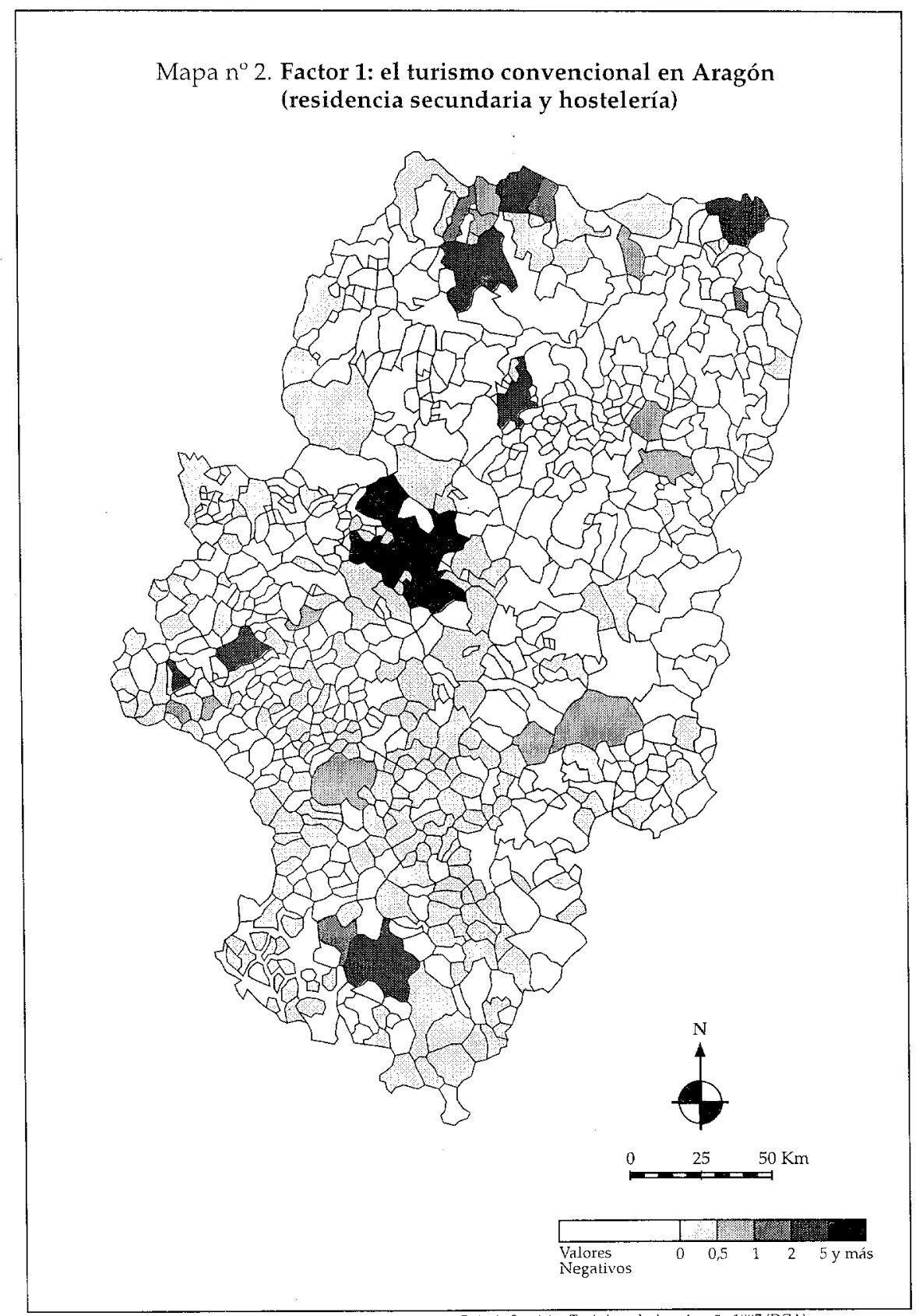

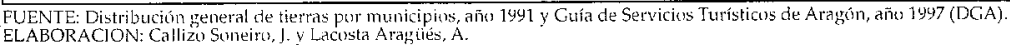


Mapa n³. Factor 2: turismo verde y turismo rural en Aragón

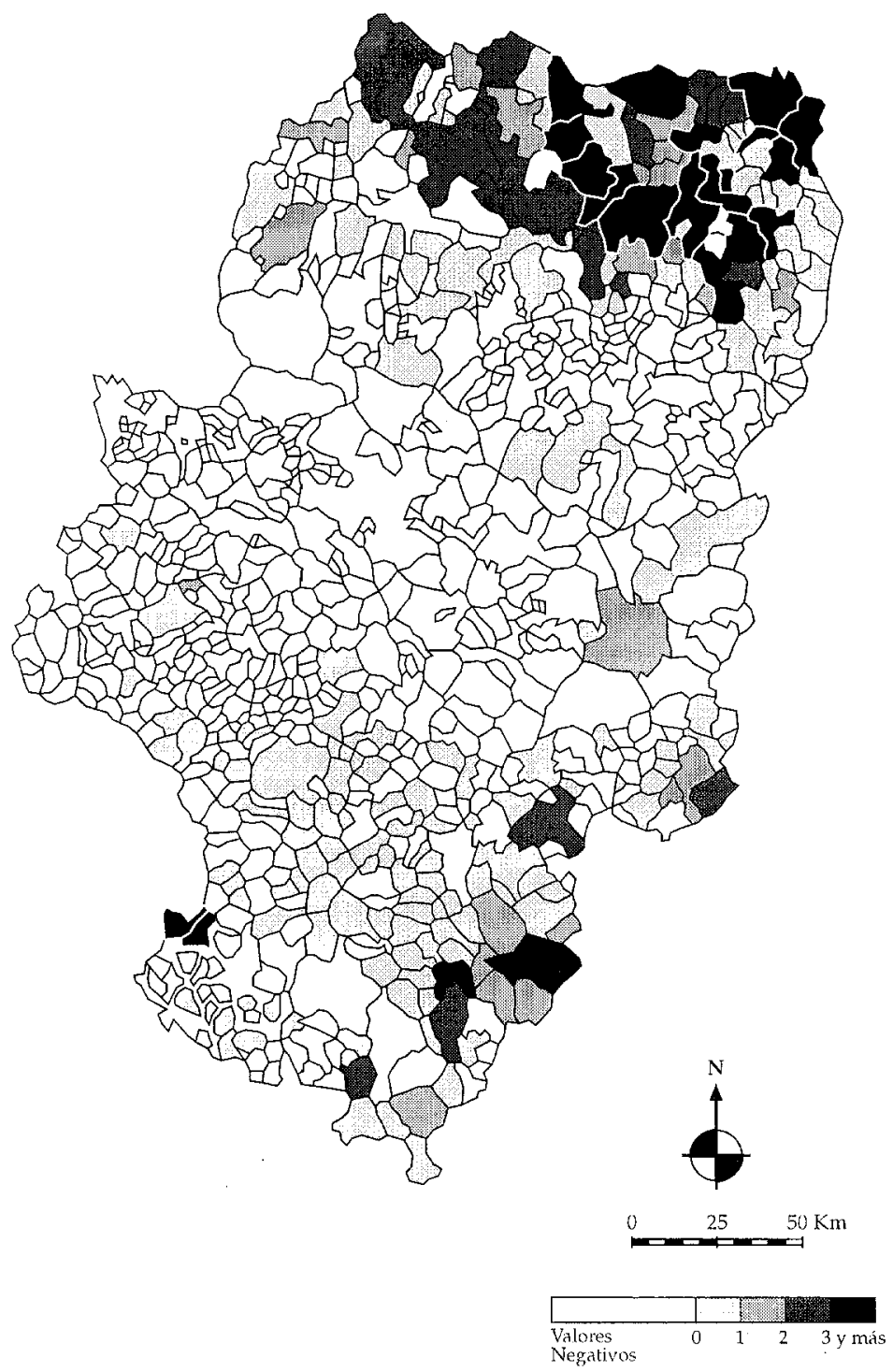

FUENTE: Distribución general de tierras por municipios, ańo 1991 y Guía de Servicios Jurísticus de Aragrón, año 1997 (DGA). 


\section{CONCLUSIONES}

1. El medio natural explica apenas el 30 por 100 de la variancia de la oferta; un porcentaje no inferior es explicable sobre todo a partir del tropismo cultural.

2. La técnica factorial prueba que, por más alta que sea la potencialidad turística de un determinado espacio natural, su cristalización como parte de la oferta no es explicable sin la decisiva intervención de los agentes económicos y sociales cuyas estrategias, actuando en etapas históricas sucesivas y en el marco de tropismos y valoraciones diferentes, están detrás de la parte no explicada de la variancia.

3. El análisis multivariante prueba también que el conjunto de actividades recreativas relacionadas con el turismo "en" el medio rural tiene una elevada relación de dependencia con las variables del medio natural; mucho mayor desde luego que la que muestran los productos clásicos.

4. La bondad del modelo de Warszynska es más evidente si se trata de explicar la potencialidad de la oferta de turismo verde y rural que si de predecir la localización de la oferta convencional.

\section{BIBLIOGRAFÍA}

CLAUSSE, R. et GUEROUT, A. (1955): "La durée des précipitations, indice climatique ou élément de climatologie touristique", La Météorologie, 37, 1-9.

DAUPHINE, A et GHILARDI, N. (1978): "Essai de bioclimatologie touristique: la Côte d'Azur", Méditerranée, 3, 3-15.

DAVIES, W.K. (1967): "Centrality and the Central Place Hierarchy", Urban Studies, 4, 67-69.

DAVIS, N.E. (1968): "An optimum summer weather index", Weather, t. XXIII, 8, 305-317.

FLOCAS, A.A. (1975): "Winter and summer indices in Athens", Scientific Anuals of the Faculty of Physics and Mathematics, Aristolelian University of Thessalonki, t. XV, 247-264.

HUGHES, G.H. (1967): "Summers in Manchester", Weather, t. XXII, 5, 199-200.

MARCHAND, J.P. (1986): "Tourisme et contraintes climatiques. L'exemple irlandais", Bulletin de l'Association de Géographes Français, t. LXIII, 5, 269-374. 
MIECZKOWSKI, Z. (1983): "The feasibility and necessity of climatic classification for purposes of tourism", Procedings. International Geographical Union Conmission for Tourisn and Leisure, Lodz Symposinm, 315-331.

MIECZKOWSKI, Z. (1985): "The tourism climatic index: a method of evaluating world climates for tourism", The Canadian Geographer/Le Géographe canadien, t. XXIX, 3, 220-233.

SARRAMEA, J. (1980): "Un indice climatico-touristique pour quelques stations balnéaires françaises", Annales de Géographie, t. LXXXIX, 495, 588-604.

SARRAMEA, J. (1981): "Estimation de la fréquentation touristique d'une station (deux éléments d’enquêtes appliquées à Fréjus-Saint Raphaël)", Méditerranée, 1, 39-46.

WARSZYNSKA, J. (1974): "Ocena zasobów srodowiska naturalnego dla potrzeb turystyki (na przykladzie Woj. Krakowskiego)" (An evaluation of natural environment resources for the development of tourist functions. As exemplified by the Cracow province), Prace Geograficzute, z. 36. 